TITLE:

\title{
Ultra-small-angle neutron scattering studies on phase separation of poly(vinyl alcohol) gels
}

\section{$\operatorname{AUTHOR}(\mathrm{S}):$}

Takeshita, H; Kanaya, T; Nishida, K; Kaji, K;

Takahashi, T; Hashimoto, M

\section{CITATION:}

Takeshita, $H$...[et al]. Ultra-small-angle neutron scattering studies on phase separation of poly(vinyl alcohol) gels. Physical Review E 2000, 61(2): 2125-2128

\section{ISSUE DATE:}

2000-02

URL:

http://hdl.handle.net/2433/39898

RIGHT:

Copyright 2000 American Physical Society 


\title{
Ultra-small-angle neutron scattering studies on phase separation of poly(vinyl alcohol) gels
}

\author{
H. Takeshita, T. Kanaya,* K. Nishida, and K. Kaji \\ Institute for Chemical Research, Kyoto University, Uji, Kyoto-fu 611-0011, Japan \\ T. Takahashi and M. Hashimoto \\ Institute for Solid State Physics, The University of Tokyo, Roppongi, Minato-ku, Tokyo 106-0032, Japan
}

(Received 20 July 1999)

\begin{abstract}
Time-resolved light scattering measurements during the gelation process of a poly(vinyl alcohol) (PVA) solution in a mixture of dimethyl sulfoxide and water $\left(\mathrm{H}_{2} \mathrm{O}\right)$ have shown that a spinodal decomposition (SD) type phase separation takes place in the early stage of gelation. In this case the kinetics of SD is valid only before macroscopic gelation occurs because the growth rate is slowed down by gelation. Such SD type phase separation makes the solution opaque as it proceeds, and hence the structural change can no longer be followed by light scattering. To investigate the structure of the opaque PVA gel as well, we have employed an ultrasmall-angle neutron scattering technique using a Bonse-Hart camera. These observations reveal that even after the macroscopic gelation the structure due to the microphase separation on a spatial scale of several $\mu \mathrm{m}$ continues to grow against the elasticity. This may be because at first the gel structure is too soft to suppress the growth of the microphase separation, but within $24 \mathrm{~h}$ after the quenching the growth terminates. On the basis of the results, we will discuss a possible mechanism of the microphase separation after gelation.
\end{abstract}

PACS number(s): 61.12.Ex, 82.70.Gg, 64.75.+g

\section{INTRODUCTION}

Polymer gels, three-dimensional networks of polymer chains, are classified into chemical and physical gels according to the types of crosslinking points or junction points [1]. The former gels are crosslinked by chemical bonds and the latter ones are formed by physical crosslinking points such as multiple (double or triple) helix strands for biopolymers, microcrystallites for crystalline polymers, and nodules for ABA type block copolymers. Most of these physical gels are thermoreversible; such a homogeneous solution at a high temperature becomes a gel upon cooling and it alternatively becomes sol upon heating. In some cases, the gelation process is accompanied by liquid-liquid phase separation as a result that the system enters the unstable state [2-4]. The structures of such gels are more complicated because they are determined by competition between phase separation and gelation $[5,6]$.

In the case of the gelation of poly(vinyl alcohol) (PVA) in a mixture of dimethyl sulfoxide (DMSO) and water with a volume ratio 60/40, a spinodal decomposition (SD) type microphase separation was expected from the macroscopic solgel diagram and the turbidity of system [5], which was directly confirmed from time-resolved light scattering (LS) measurements in the early stage of gelation [7]. However, LS can be used to study only the very early stage of phase separation because the turbidity of the solution increases as the phase separation proceeds.

For investigation of the structure of opaque materials, neutron and $\mathrm{x}$-ray scattering techniques are useful. Conventional small-angle neutron scattering (SANS) or x-ray scattering cameras can access a range of $\sim 0.003$ to $\sim 0.2 \AA^{-1}$ in

\footnotetext{
*Author to whom correspondence should be addressed. Electronic address: kanaya@scl.kyoto-u.ac.jp
}

$Q$ (length of scattering vector), corresponding to $\sim 30$ $\sim 2000 \AA$ on a spatial scale $(2 \pi / Q)$ while ultra-small-angle neutron scattering (USANS) and ultra-small-angle x-ray scattering cameras of a Bonse-Hart type [8-10] cover $Q$ values ranging from approximately $10^{-5}$ to $10^{-2} \AA^{-1}$, which include the $Q$ range of LS. In this work, we have employed the USANS technique to investigate the structure of the opaque PVA gels to elucidate the mechanism of microphase separation after macroscopic gelation. Time-resolved LS measurements were also extended to PVA solutions with concentrations 2 and $10 \mathrm{~g} / \mathrm{dL}$ in addition to the previously reported $5 \mathrm{~g} / \mathrm{dL}$ solution [7].

\section{EXPERIMENT}

Fully saponified atactic poly(vinyl alcohol) (PVA) with a number-average degree of polymerization $P=1730$ was used in this work. The molecular weight distribution $M_{w} / M_{n}$ is 1.97 where $M_{w}$ and $M_{n}$ are weight- and number-average molecular weights, respectively. The details of the characterization of this sample and the procedure of gel preparation were reported in previous papers $[5,11,12]$. In order to avoid strong incoherent scattering from hydrogen atoms a deuterated solvent was used for the USANS measurements: a mixture of deuterated dimethyl sulfoxide (DMSO- $d_{6}$ ) and heavy water $\left(\mathrm{D}_{2} \mathrm{O}\right)$ with a volume ratio $60 / 40$ while a mixture of their protonated ones was used for the LS measurements. The temperature of the samples was controlled to be $25^{\circ} \mathrm{C} \pm 0.1^{\circ} \mathrm{C}$ during the measurements.

USANS measurements were carried out using the ULS spectrometer [13] with a Bonse-Hart camera installed at the cold neutron source of the JRR-3M reactor, Tokai. The wavelength of incident neutrons was $4.73 \AA$. The full width at half maximum of the rocking curve was $4.4 \mathrm{~s}$ for the $\mathrm{Si}$ 111 channel-cut single crystal with five successive reflections. This spectrometer covers a range $10^{-5}-10^{-2} \AA^{-1}$ in 


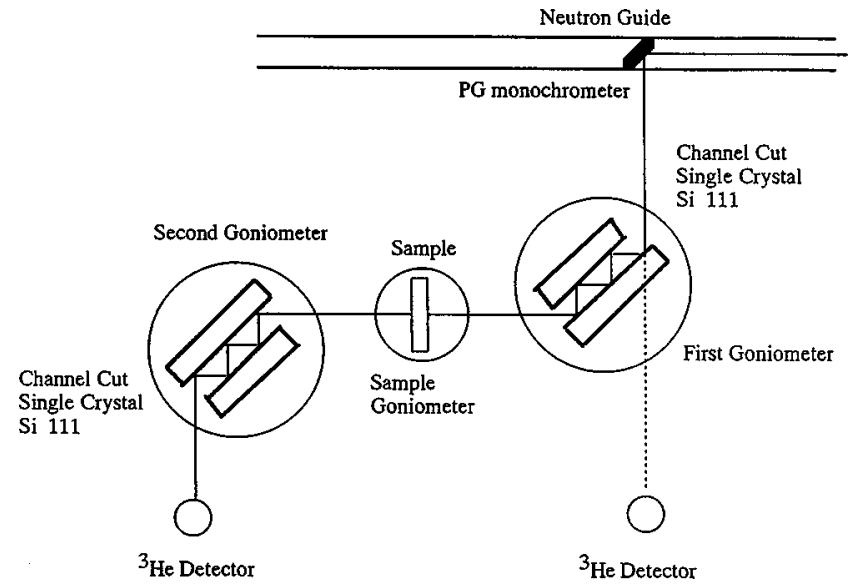

FIG. 1. Geometry of the ultra-small-angle neutron scattering spectrometer ULS at the JRR-3M reactor, Tokai.

length of scattering vector $(Q=4 \pi \sin \Theta / \lambda)$. The geometry of the spectrometer is schematically shown in Fig. 1.

LS measurements were carried out with a System 4700 of Malvern Instruments Inc. using an $\mathrm{Ar}^{+}$laser $(\lambda$ $=488 \mathrm{~nm}, 75 \mathrm{~mW}$ ) as a light source. The covering range of LS in length of scattering vector $Q=4 \pi n \sin \Theta / \lambda, n$ being the refractive index, is $3.6 \times 10^{-4}$ to $3.4 \times 10^{-3} \AA^{-1}$. In order to reduce the effects of inhomogeneity (speckle) arising from the gel structure itself, the measurements were performed by rotating a sample cell $(10 \mathrm{~mm} \phi)$.

\section{RESULTS AND DISCUSSION}

PVA solutions in the $60 / 40\left(\mathrm{DMSO} / \mathrm{H}_{2} \mathrm{O}\right)$ mixture were homogenized at $100{ }^{\circ} \mathrm{C}$ and quenched to $25^{\circ} \mathrm{C}$ for gelation. Just after the quenching, we started time-resolved LS measurements on the solution. Figure 2 shows such an example of the time evolution of the scattering intensity $I(Q)$ for the solution of $5 \mathrm{~g} / \mathrm{dL}$. In this figure a single broad peak of the scattering intensity $I(Q)$ is observed and its peak intensity

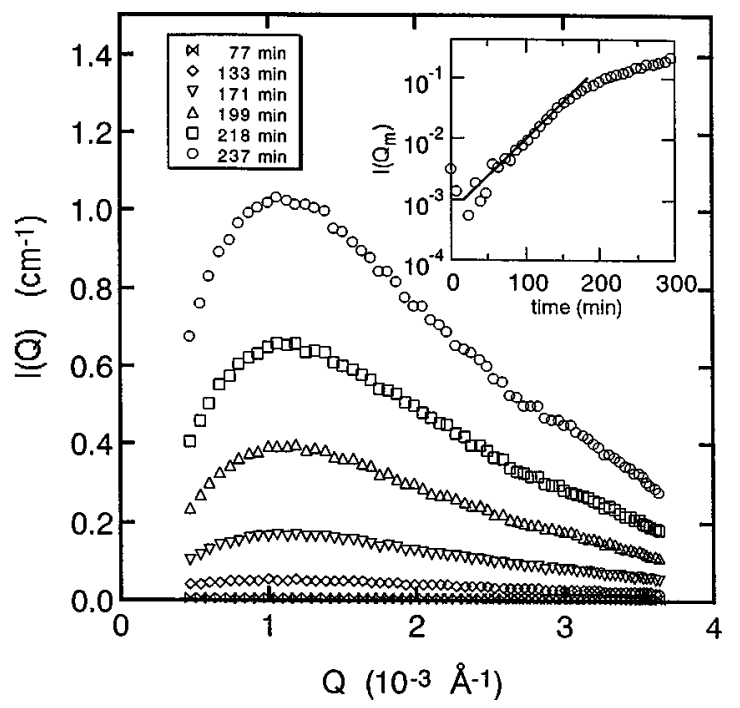

FIG. 2. Time evolution of light scattering intensity $I(Q)$ for a PVA solution $\left(C_{p}=5 \mathrm{~g} / \mathrm{dL}\right)$ at $25^{\circ} \mathrm{C}$ after a temperature jump from $100{ }^{\circ} \mathrm{C}$. The inset shows the time evolution of the scattering intensity at the maximum position $I\left(Q_{m}\right)$ in a semilogarithmic form. increases with time. Similar results were obtained for solutions of 2 and $10 \mathrm{~g} / \mathrm{dL}$. The maximum positions $Q_{m}$ of such peaks for the concentrations 2,5 , and $10 \mathrm{~g} / \mathrm{dL}$ were 1.1 $\times 10^{-3}, 1.2 \times 10^{-3}$, and $1.25 \times 10^{-3} \AA^{-1}$, corresponding to characteristic wavelengths $\Lambda_{c}=0.57,0.52$, and $0.50 \mu \mathrm{m}$, respectively. These maximum positions $Q_{m}$ were almost constant independent of time until about $240 \mathrm{~min}$. In the inset of Fig. 2, the scattering intensity at the maximum position $I\left(Q_{m}\right)$ is plotted as a function of time in a semi-logarithmic form. The intensity $I\left(Q_{m}\right)$ increases exponentially with time up to about $160 \mathrm{~min}$, but thereafter it levels off. Here, it should also be noted that slowing-down of the growth rate of the scattering intensity at $Q_{m}$ is not due to the transition from the early stage to the late stage in phase separation. This is because the macroscopic gelation time $t_{g}$ determined by a ball-falling method [11] was $180 \mathrm{~min}$ for the $5 \mathrm{~g} / \mathrm{dL}$ solution, which corresponds almost to the deceleration point, and as will be shown later, the characteristic wavelength obtained from $Q_{m}$ does not change yet at around $t_{g}$ in spite of the fact that the scattering peak intensity $I\left(Q_{m}\right)$ continues to increase. Thus, the system still remains in the early stage at around the gelation time. From these two facts the slowingdown of the scattering intensity $I(Q)$ seems to be due to brake effect by gelation.

The experimental results of Fig. 2 agree with the predictions from the linearized theory for the early stage of spinodal decomposition (SD) type phase separation presented by Cahn [14], confirming that as the PVA solution is quenched to $25^{\circ} \mathrm{C}$, the $\mathrm{SD}$ type microphase separation initiates before the macroscopic gelation.

In this system, the gelation occurs through the network formation within the concentrated (polymer rich) phase of the bicontinuous structure caused by the SD type microphase separation [5,7], where the crosslinking points are crystallites [15]. At the gelation time $t_{g}$, the PVA solution loses fluidity. Nevertheless, the microscopic structure probed by the LS still continues to grow even after macroscopic gelation as seen in the inset of Fig. 2 although the growth rate of $I\left(Q_{m}\right)$ slows down at around the gelation time $t_{g}(=180 \mathrm{~min})$. As the structural growth proceeds, the PVA gel becomes opaque, and hence the structural evolution can no longer be followed by LS. We therefore employed a USANS technique for the study of the opaque PVA gels.

In the USANS measurements, it takes about $12 \mathrm{~h}$ to obtain one spectrum for the PVA gels. Just after the temperature jump of a homogeneous solution from $100^{\circ} \mathrm{C}$ to $25^{\circ} \mathrm{C}$, the USANS measurements were performed every $12 \mathrm{~h}$; it took three days to take six spectra for a given concentration. It was found that the first two spectra measured between 0 and $12 \mathrm{~h}$ and between 12 and $24 \mathrm{~h}$ are time dependent while the third-to-sixth spectra are identical with one another within experimental error, suggesting that the structural evolution due to the microphase separation in the gel terminates at least within $24 \mathrm{~h}$ after the quenching [16]. In order to obtain higher counting statistics the latter four timeindependent spectra, measured between 24 and $72 \mathrm{~h}$ after the quenching, were added together. Figure 3 shows such results for the solutions of 2, 5, and $10 \mathrm{~g} / \mathrm{dL}$ and the solvent as well as the resolution function (the rocking curve) for reference. Further, these spectra, from which the solvent spectrum was subtracted, were desmeared for the broadening due to the 


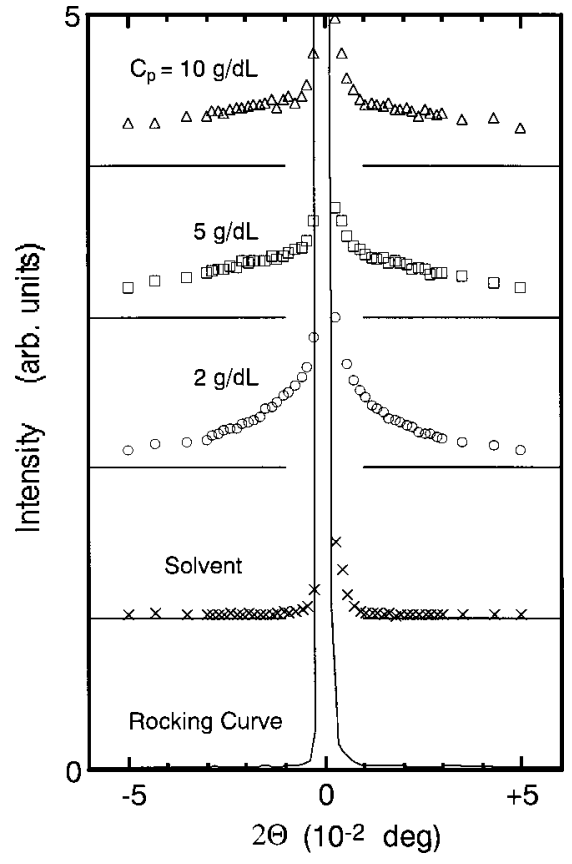

FIG. 3. Observed USANS spectra for PVA solutions with $C_{p}$ $=2$, 5, and $10 \mathrm{~g} / \mathrm{dL}$. For reference, the spectra of the solvent (DMSO $-d_{6} / \mathrm{D}_{2} \mathrm{O}, 60 / 40$ in volume) and the resolution (rocking curve) are also indicated.

line profile of collimation slits.

The desmeared spectra $I_{d s}(Q)$ normalized with the polymer concentration $C_{p}$ are shown in Fig. 4 for the 2, 5, and 10 $\mathrm{g} / \mathrm{dL}$ solutions and those expanded by a factor 5 are seen in the inset. For the 5 and $10 \mathrm{~g} / \mathrm{dL}$ solutions, $I_{d s}(Q)$ indicates a broad maximum at $Q_{m}=3.9 \times 10^{-4}$ and $4.5 \times 10^{-4} \AA^{-1}$, giving characteristic wavelengths $\Lambda_{c}$ of 1.6 and $1.4 \mu \mathrm{m}$, respectively. These $\Lambda_{c}$ 's are plotted against time in Fig. 5, where $\Lambda_{c}$ values in the early stage of phase separation determined by the LS are also included. For the 5 and $10 \mathrm{~g} / \mathrm{dL}$ solutions, the $\Lambda_{c}$ values after $24 \mathrm{~h}$ are 3.1 and 2.8 times larger than those in the early stage before $5 \mathrm{~h}$, respectively, suggesting that the structure caused by the microphase separation considerably grows in size with time even after gela-

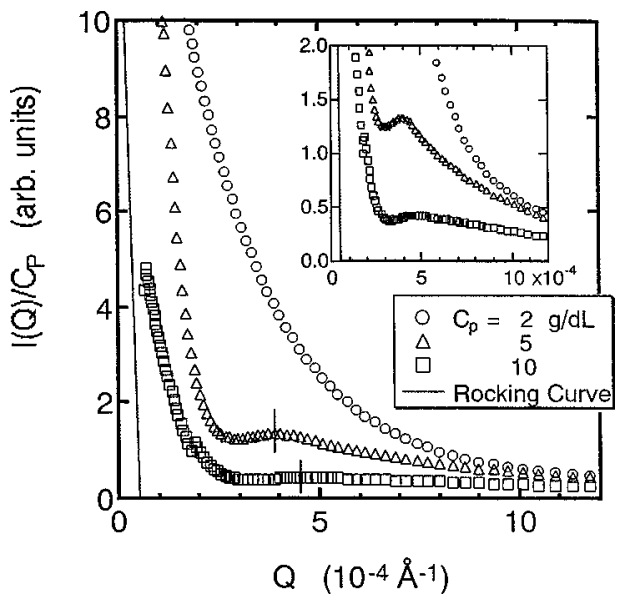

FIG. 4. Desmeared USANS spectra for PVA solutions after subtracting the solvent scattering. In the inset the spectra are expanded by a factor 5 .

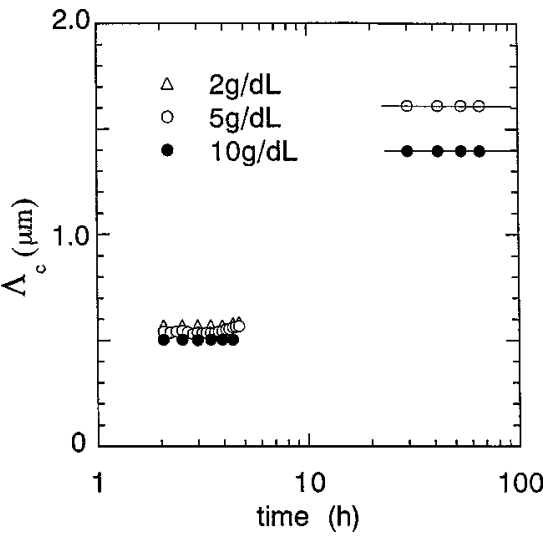

FIG. 5. Time evolution of characteristic wavelength $\Lambda_{c}$ for PVA solutions with concentrations 2,5 , and $10 \mathrm{~g} / \mathrm{dL}$. The data before $5 \mathrm{~h}$ were measured by light scattering.

tion. As has already been described for the $5 \mathrm{~g} / \mathrm{dL}$ solution in Fig. 2, until 160 min the maximum position $Q_{m}$ did not change with time and the peak intensity increased exponentially with time, and hence this period corresponds to the early stage of SD. Macroscopic gelation occurred at around $180 \mathrm{~min}\left(t_{g}=3.0 \mathrm{~h}\right)$, but if the gelation does not occur, the structural growth would proceed via coalescence of the bicontinuous phase to reduce the interfacial energy, resulting in an increase of the characteristic wavelength as observed in microphase separation of polymer blends [17]. In this case the kinetics would be described by the scaling theory presented by Furukawa [18], predicting that the characteristic wavelength $\Lambda_{c}$ and the scattering intensity $I\left(Q_{m}\right)$ increase with time according to the power laws $\Lambda_{c} \sim t^{a}$ and $I\left(Q_{m}\right)$ $\sim t^{b}$, respectively, and the ratio $b / a$ corresponds to the dimensionality of the system $d(=3)$. However, because of the gelation the kinetics of the phase separation does not agree with the theoretical prediction, which seems to be very natural [19]. Nevertheless we can consider that at around $4.0 \mathrm{~h}$ when $\Lambda_{c}$ begins to increase (see Fig. 5) the phase separation process enters the late stage. What we have to emphasize here is that the gel structure just after gelation is too soft to suppress the structural growth through the coalescence. This may be not only because the concentration in the dilute (polymer poor) phase is very low, but also because the crosslinking density in the concentrated (polymer rich) phase is very small just after gelation, and hence the coalescence of the bicontinuous phase becomes possible against the elasticity of the gel network, resulting in the increase of the characteristic wavelength $\Lambda_{c}$.

As seen from Fig. 4, the scattering intensity $I_{d s}(Q)$ has no peak for the $2 \mathrm{~g} / \mathrm{dL}$ solution, meaning that the characteristic wavelength $\Lambda_{c}$ is out of the $Q$ range or has disappeared. In the $2 \mathrm{~g} / \mathrm{dL}$ solution, the fraction of concentrated phase after the microphase separation is much less than those in the 5 and $10 \mathrm{~g} / \mathrm{dL}$ solutions, so that the elasticity of the system is lower. Hence the microscopic structure due to the SD type phase separation grows much faster in the $2 \mathrm{~g} / \mathrm{dL}$ solution than in the 5 and $10 \mathrm{~g} / \mathrm{dL}$ solutions. On this basis we expect that the characteristic wavelength of the $2 \mathrm{~g} / \mathrm{dL}$ gel presumably exists in a lower $Q$ range than examined.

In Fig. 4 it is also worth pointing out that $I_{d s}(Q)$ for the 5 and $10 \mathrm{~g} / \mathrm{dL}$ solutions increases with decreasing $Q$ below 
$Q=\sim 3.0 \times 10^{-4} \AA^{-1}$, corresponding to more than $\sim 2 \mu \mathrm{m}$ $[=2 \pi / Q]$ on a spatial scale. This upturn is not observed in the early stage of phase separation, implying that some structure larger than $\sim 2 \mu \mathrm{m}$ is formed after the macroscopic gelation in addition to the bicontinuous structure giving a characteristic wavelength $\Lambda_{c}$. Although the origin of the upturn is not well understood at present, two possibilities are considered; one is deformation of the bicontinuous network of the concentrated phase in which polymer chains are crosslinked, and the other is production of so-called isolated microgels which are observed in PVA solutions with a concentration lower than the critical gelation concentration $C_{g} *$ $(=1.35 \mathrm{~g} / \mathrm{dL})[15]$.

\section{CONCLUSION}

The LS studies revealed that the spinodal decomposition (SD) type phase separation takes place in PVA solutions in a $60 / 40$ (DMSO- $d_{6} / \mathrm{D}_{2} \mathrm{O}$ ) mixture before the macroscopic gelation. After the gelation the scattering intensity still in- creases with time, suggesting that the microscopic structure grows against the elasticity in the gel. Such structural evolution can no longer be followed by the LS because the gel is opaque. We therefore employed USANS technique. It was shown by the USANS study on the opaque gels that a maximum is observed in the scattering profile $I_{d s}(Q)$ for the 5 and $10 \mathrm{~g} / \mathrm{dL}$ gels, giving characteristic wavelengths $\Lambda_{c}$ $=1.6$ and $1.4 \mu \mathrm{m}$, respectively, which are 3.1 and 2.8 times larger than those in the early stage. This suggests that the microscopic structure due to phase separation grows in size even after the macroscopic gelation. In the $2 \mathrm{~g} / \mathrm{dL}$ gel, on the other hand, no peaks were observed in the scattering profile $I_{d s}(Q)$. This was interpreted in terms of the lower elasticity of the $2 \mathrm{~g} / \mathrm{dL}$ gel. In the very low $Q$ range below 3.0 $\times 10^{-4} \AA^{-1}$ the upturn of scattering intensity was observed for the 5 and $10 \mathrm{~g} / \mathrm{dL}$ gels though it was not detected in the early stage of the gelation, suggesting that very large structure is produced in the late stage of microphase separation process in the gels.
[1] P. G. d. Gennes, Scaling Concepts in Polymer Physics (Cornell University Press, Ithaca, 1979).

[2] E. Pines and W. Prins, Macromolecules 6, 888 (1973).

[3] M. Komatsu, T. Inoue, and K. Miyasaka, J. Polym. Sci., Polym. Phys. Ed. 24, 303 (1986).

[4] C. Hara and M. Matsuo, Polymer 36, 603 (1995).

[5] M. Ohkura, T. Kanaya, and K. Kaji, Polymer 33, 3689 (1992).

[6] T. Kanaya, H. Takeshita, Y. Nishikoji, M. Ohkura, K. Nishida, and K. Kaji, Supramol. Sci. 5, 215 (1998).

[7] H. Takeshita, T. Kanaya, K. Nishida, and K. Kaji, Macromolecules 32, 7815 (1999).

[8] U. Bonse and M. Hart, Appl. Phys. Lett. 7, 238 (1965).

[9] U. Bonse and M. Hart, Z. Phys. 189, 151 (1965).

[10] U. Bonse and M. Hart, in Small-Angle X-ray Scattering, edited by H. Brumberger (Gordon and Breach, New York, 1965), p. 121.
[11] M. Ohkura, T. Kanaya, and K. Kaji, Polymer 33, 5044 (1992).

[12] T. Kanaya, M. Ohkura, H. Takeshita, K. Kaji, M. Furusaka, H. Yamaoka, and G. D. Wignall, Macromolecules 28, 3168 (1995).

[13] T. Takahashi, Activity Report on Neutron Scattering Research, ISSP, University of Tokyo, No. 1, 1994, p. 34 (unpublished).

[14] J. W. Cahn, J. Chem. Phys. 42, 93 (1965).

[15] T. Kanaya, M. Ohkura, K. Kaji, M. Furusaka, and M. Misawa, Macromolecules 24, 5609 (1994).

[16] It is noted that very slow volume change is observed during several months. However, this change is too slow to affect the present results.

[17] T. Izumitani, M. Takenaka, and T. Hashimoto, J. Chem. Phys. 92, 3213 (1990).

[18] H. Furukawa, Adv. Phys. 34, 703 (1985).

[19] A. Onuki and S. Puri, Phys. Rev. E 59, 1331 (1999). 